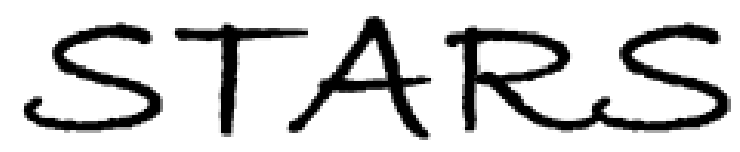

University of Central Florida

STARS

$1-1-2011$

\title{
Plume Development of The Shoemaker-Levy 9 Comet Impact
}

\author{
Csaba Palotai \\ University of Central Florida \\ Donald G. Korycansky \\ Joseph Harrington \\ University of Central Florida \\ Noémi Rebeli \\ University of Central Florida \\ Travis Gabriel \\ University of Central Florida
}

Find similar works at: https://stars.library.ucf.edu/facultybib2010 University of Central Florida Libraries http://library.ucf.edu

This Article is brought to you for free and open access by the Faculty Bibliography at STARS. It has been accepted for inclusion in Faculty Bibliography 2010 s by an authorized administrator of STARS. For more information, please contact STARS@ucf.edu.

\section{Recommended Citation}

Palotai, Csaba; Korycansky, Donald G.; Harrington, Joseph; Rebeli, Noémi; and Gabriel, Travis, "Plume Development of The Shoemaker-Levy 9 Comet Impact" (2011). Faculty Bibliography 2010s. 1743. https://stars.library.ucf.edu/facultybib2010/1743

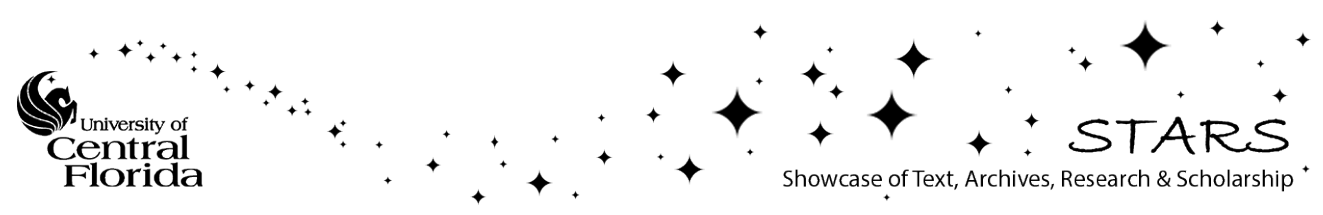




\title{
PLUME DEVELOPMENT OF THE SHOEMAKER-LEVY 9 COMET IMPACT
}

\author{
Csaba Palotai ${ }^{1}$, Donald G. Korycansky ${ }^{2}$, Joseph Harrington ${ }^{1}$, NoÉmi Rebeli ${ }^{1}$, And Travis Gabriel ${ }^{1}$ \\ ${ }^{1}$ Planetary Sciences Group, Department of Physics, University of Central Florida, Orlando, FL 32816-2385, USA; csaba@physics.ucf.edu \\ ${ }^{2}$ Department of Earth and Planetary Sciences, University of California, Santa Cruz, CA 95064, USA \\ Received 2010 November 22; accepted 2011 January 18; published 2011 March 16
}

\begin{abstract}
We have studied the plume formation after a Jovian comet impact using the ZEUS-MP 2 hydrodynamics code. The three-dimensional models followed objects with 500, 750, and $1000 \mathrm{~m}$ diameters. Our simulations show the development of a fast, upward-moving component of the plume in the wake of the impacting comet that "pinches off" from the bulk of the cometary material $\sim 50 \mathrm{~km}$ below the 1 bar pressure level, $\sim 100 \mathrm{~km}$ above the depth of the greatest mass and energy deposition. The fast-moving component contains about twice the mass of the initial comet, but consists almost entirely $(>99.9 \%)$ of Jovian atmosphere rather than cometary material. The ejecta rise mainly along the impact trajectory, but an additional vertical velocity component due to buoyancy establishes itself within seconds of impact, leading to an asymmetry in the ejecta with respect to the entry trajectory. The mass of the upward-moving component follows a velocity distribution $M(>v)$ approximately proportional to $v^{-1.4}\left(v^{-1.6}\right.$ for the $750 \mathrm{~m}$ and $500 \mathrm{~m}$ cases) in the velocity range $0.1 \mathrm{~km} \mathrm{~s}^{-1}<v<10 \mathrm{~km} \mathrm{~s}^{-1}$.
\end{abstract}

Key words: comets: individual (Shoemaker-Levy 9) - hydrodynamics - methods: numerical - shock waves

Online-only material: color figures

\section{INTRODUCTION}

In 1994 July, several fragments of comet Shoemaker-Levy 9 (SL9) impacted Jupiter, giving a unique opportunity for direct observation of a hypervelocity impact and its aftermath. The scientific community labeled it as "once in a lifetime event" because at that point the estimates for the probability of a similar event taking place suggested that decades or centuries might pass before it happened again. The 2009 July 19 impact discovered by A. Wesley (Hammel et al. 2010; Sánchez-Lavega et al. 2010) and the 2010 June 3 impact discovered by C. Go and A. Wesley (Hueso et al. 2010) indicate that Jupiter impacts happen much more frequently.

Harrington et al. (2004) give an overview of the phenomenology common to all the larger impacts. In the short but energetic entry phase, the SL9 fragments entered Jupiter's atmosphere at $\sim 45^{\circ} \mathrm{S}$ latitude with an impact velocity of over $60 \mathrm{~km} \mathrm{~s}^{-1}$ and an impact angle of about $45^{\circ}$ (Chodas \& Yeomans 1996). During entry, the impactors broke up and evaporated in $\sim 10$ s (Korycansky et al. 2006), depositing most of their kinetic energy close to the terminal depth. Each impact created a lowdensity entry channel consisting of high-temperature Jovian air and impactor material (Mac Low \& Zahnle 1994; Zahnle \& Mac Low 1994, 1995; Crawford et al. 1995; Korycansky et al. 2006). This column of debris rose back up in the entry channel and expanded radially, generating shock waves. At higher altitudes, the plume rose ballistically, with the visible top of the debris reaching about $3000 \mathrm{~km}$ above the cloud tops (Hammel et al. 1995; Jessup et al. 2000). The plume then collapsed, compressing and heating itself and the upper atmosphere it encountered as it continued to expand radially (Deming \& Harrington 2001).

Modeling the observed phenomena is difficult because the various stages of the process have time and length scales that differ by several orders of magnitude and the relevant physics and chemistry cannot be incorporated into a single model. Instead, modelers have simulated individual phases of the impact. Initial conditions for models of later phases (entry response, plume flight, plume splash) come from remapping the final state of the previous phase onto a larger grid. Zahnle \& Mac Low (1994) and Crawford et al. (1995) chained the entry and the blowout phases in this manner, while Harrington \& Deming (2001) and Deming \& Harrington (2001) connected the plume flight and landing response phases using the same technique in a two-dimensional (2D) model. Exceptions to this practice are Takata et al. (1994) and Takata \& Ahrens (1997), who used a smoothed particle hydrodynamics (SPH) model to include both the entry and the entry-response phases in their three-dimensional (3D) simulations. Harrington et al. (2004) give a more complete review of previous modeling efforts.

Since all subsequent phases depend on the entry, Korycansky et al. (2006) performed high-resolution, 3D simulations, computing energy deposition profiles and terminal depths of various impactor types. In this paper, our investigation focuses on plume development in the immediate aftermath of an SL9 comet fragment's entry.

\section{MODEL DESCRIPTION}

We modified the ZEUS-MP 2 hydrodynamics code (Hayes et al. 2006) for Jovian impacts, validating the modifications with a series of tests. These included ZEUS-MP's own hydrodynamic test suite (e.g., Sedov-Taylor blast wave, radiating shock waves) and our results matched the published data (Hayes et al. 2006). We also set up simulations to verify hydrostatic equilibrium and the long-term stability of the model Jovian atmosphere over many sound-crossing times. Past work on atmospheric impacts (Korycansky et al. 2002) found significant differences in results between 2D and 3D simulations. In particular, geometrical constraints that operate in two dimensions (but not in three) tend to enforce global enstrophy conservation, which in turn forces a portion of the flow kinetic energy into large scales. Twodimensional calculations thus show unrealistic amounts of largescale structure (Khokhlov 1994). Because of the qualitative differences between 2D and 3D fluid dynamics, 3D models provide more accurate results than those of the SL9 era. 

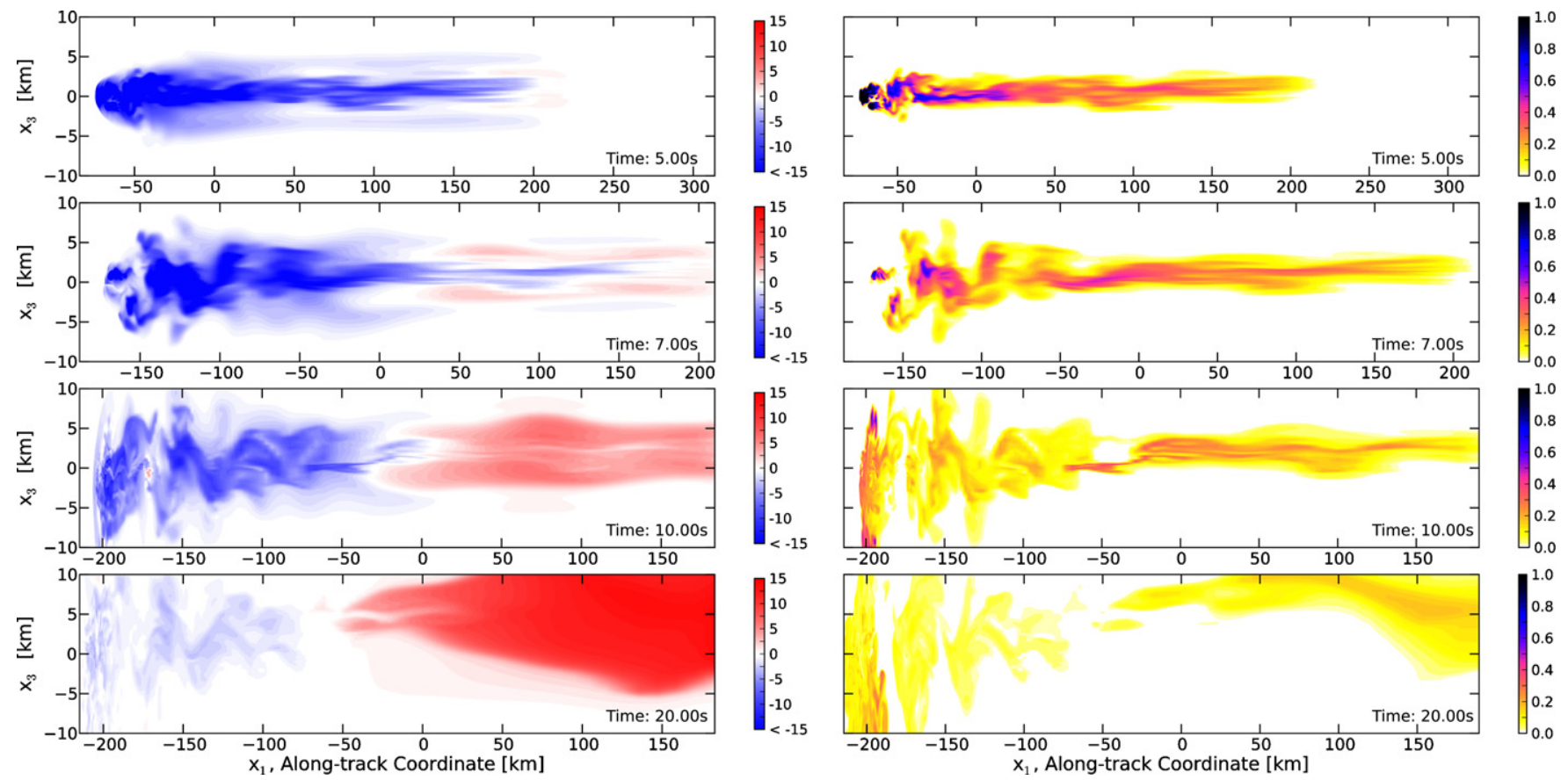

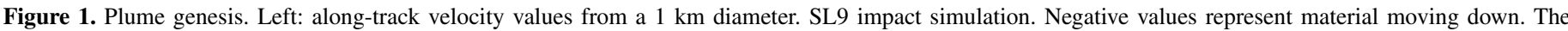

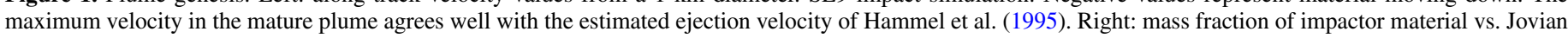
air in the same simulation. Note the position of the origin.

(A color version of this figure is available in the online journal.)

In order to delay the remapping of variables to a second model as long as possible, we studied the plume's genesis and its effect on the Jovian atmosphere by extending the grid of Korycansky et al. (2006) and continuing calculations beyond the $\sim 10 \mathrm{~s}$ of impactor destruction. The sensitivity tests of Korycansky et al. (2006) determined that having a higher resolution than 16 grid elements within the impactor's radius $(R 16)$ did not result in significant changes in the impactor's disruption mode or penetration depth, so we used $R 16$ for the present models. The Cartesian coordinate system aligns with the initial trajectory of the impactor; $x_{1}$ is the along-track coordinate, $x_{2}$ is horizontal, and $x_{3}$ is perpendicular to the others. The 1 bar pressure level coincides with the origin of the $x_{1}$ coordinate. Away from the high-resolution inner region that contains the impactor, the spacing increases by $\sim 4 \%$ per grid cell. A typical grid has a size of $\sim 400 \times 90 \times 90 \mathrm{~km}$ and has $\sim 430 \times 280 \times 280$ grid elements. These numbers vary somewhat depending on the diameter of the impactor. The grid moves with the impactor to keep it in the high-resolution region and stops when the comet is vaporized. When the grid stops, there is no undisturbed Jovian air along the track of the impactor and behind it. This allows us to model the acceleration and expansion of the plume as accurately as possible. Korycansky et al. (2006) provide a more detailed description of the model.

\section{RESULTS}

Our nominal case is that of Korycansky et al. (2006), a $1 \mathrm{~km}$ diameter, $0.6 \mathrm{~g} \mathrm{~cm}^{-3}$, porous, spherical, ice impactor arriving at $44.02 \mathrm{~S}$, at $61.46 \mathrm{~km} \mathrm{~s}^{-1}$, at a 42.09 impact angle. This case is likely larger than a typical SL9 fragment (Carlson et al. 1997) but it lets us compare our results to prior work.

Figure 1 shows the impact and genesis of the plume. Initially, the shock structure behind the impactor (Figure 2) limits the distribution of cometary material to the turbulent wake, so only a narrow trail of impactor debris remains in the entry channel. The breakup of the impactor begins at about $4.2 \mathrm{~s}$ into the simulation and at about $40-50 \mathrm{~km}$ below the 1 bar pressure level (distances indicate along-track values). This event disrupts the organized flow behind the main fragment, allowing for the spreading of impactor material perpendicular to the entry path and more vigorous mixing of debris and Jovian air as the impactor descends to greater depths.

The plume forms as upward velocities appear within the entry channel. At this point we find an interesting and important property of the plume: it "pinches off" at a certain depth. Below this level, the material rises independently and much slower. Almost all of the cometary material is below this level, and although most of the energy gets deposited near the terminal depth, this material only rises buoyantly. Most likely it cannot achieve ballistic ejection (see below). The pinch-off occurs at the altitude where the breakup level of the impactor takes place. The $500 \mathrm{~m}$ and $750 \mathrm{~m}$ impactors pinch off within about a scale height of the nominal case.

Based on their 2D experiments, Boslough et al. (1995) reported that using only the energy deposited by a $3 \mathrm{~km}$ diameter impactor above the $-50 \mathrm{~km}$ level results in an almost identical fireball to that generated by using the full energy deposition curve. They concluded that the fireball growth depends mostly on the diameter of the impactor and the fact that several plumes reached the same height (Hammel et al. 1995) implies that the fragments that generated those plumes were of the same size. In our simulations the plume detachment occurs at about the same depth that Boslough et al. (1995) used for the energy deposition cutoff, which strengthens the hypothesis that the energy deposition below this level (more than $99 \%$ of the total energy deposition) would not affect the buildup of the plume. However, in our models the $1 \mathrm{~km}, 750 \mathrm{~m}$, and $500 \mathrm{~m}$ diameter impactors all broke up at similar altitudes and all of them generated ejection velocities of $>12 \mathrm{~km} \mathrm{~s}^{-1}$ despite the factor 

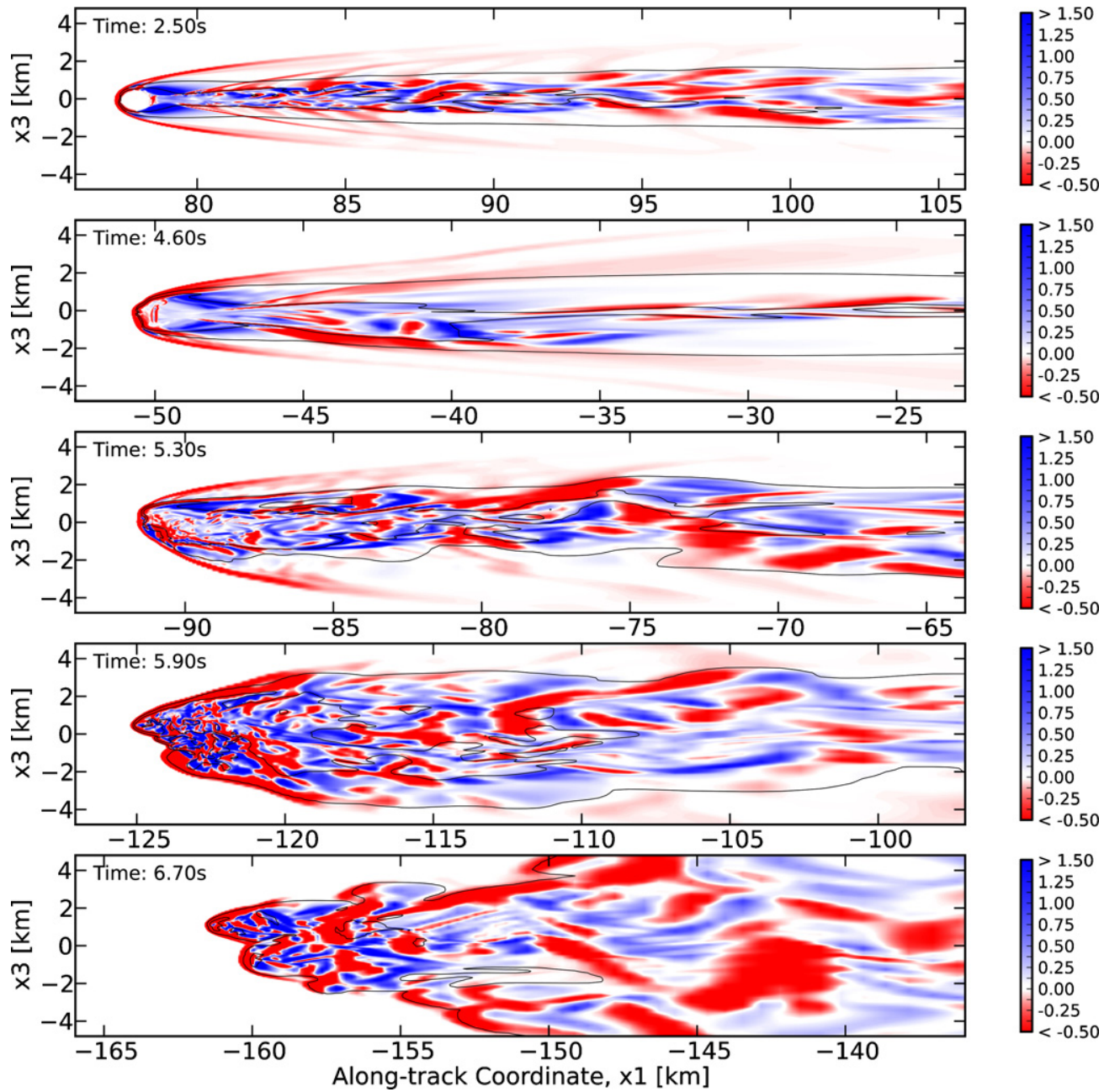

Figure 2. Shock structure and the distribution of impactor debris during the breakup of an SL9-type impactor. The color variable is $d v / d x\left(10^{9} \mathrm{~s}^{-1}\right)$. The purpose of the color is not to emphasize the variable itself but to indicate the location and the structure of the shocks. Black contour lines represent the mass fraction of comet material, the outermost line represents $1 \%$ of impactor material and the inner lines represents $80 \%$. Prior to the breakup, the shock system on the trailing edge of the impactor confines the debris that falls off of the impactor resulting in a narrow trail of cometary material within the plume. After the breakup, which occurs at about $4.2 \mathrm{~s}$, the shock system falls apart and allows for the spreading of the debris perpendicular to the entry path at greater depths.

(A color version of this figure is available in the online journal.)

of eight range of mass/kinetic energy and factor of four range in initial cross section. Thus, the observed plume heights likely could have been achieved by different-sized impactors. We plan future studies to verify this.

Crawford et al. (1995) noted that during the early stages of their model the evolving fireball maintained axisymmetry with respect to the entry channel. The oblique impact simulations of Boslough et al. (1995) and Takata et al. (1994) also predict the ejecta to rush back along the entry trajectory. In their models, Pankine \& Ingersoll (1999) aligned the axis of the ejecta cone with the trajectory of the incoming comet. Hammel et al. (1995) estimated that in order for the plume material to reach the observed height of $3000 \mathrm{~km}$ at the $45^{\circ}$ ejection angle, an initial velocity of $\sim 17 \mathrm{~km} \mathrm{~s}^{-1}$ is required for ballistic particles. However, Harrington \& Deming (2001) found that the best fit to the appearance of the impact site came with an ejection cone tilted just $30^{\circ}$ from the vertical. Our models do not produce velocities that high even for the $1 \mathrm{~km}$ diameter impactor, although it is conceivable that they may appear later as the plume blows out from the atmosphere. However, even in the first few seconds of plume formation, the entry channel expands mostly toward low pressure, creating an asymmetry with respect to the entry channel. Figure 3 shows the debris ejection directions in our model. Most of the ejecta are above the impactor's trajectory, and the deviation from the entry path increases with time. Jessup et al. (2000) determined that an initial tilt angle of $\sim 6^{\circ}-15^{\circ}$ and an initial velocity of "only" $\sim 11-12 \mathrm{~km} \mathrm{~s}^{-1}$ are required to reproduce the time-dependent behavior of the observed plume apexes from the A, E, G, and $\mathrm{W}$ impacts. We do see velocities that high in our model, even in the cases of the $750 \mathrm{~m}$ and $500 \mathrm{~m}$ diameter impactors.

Jessup et al. (2000) deduced from the Galileo Near-Infrared Mapping Spectrometer data that the $G$ fireball was most likely initiated between the 100 and 200 mbar pressure levels (35-45 km above the 1 bar level). Figure 1 shows that our model agrees with this deduction: at this level $(\sim 50-70 \mathrm{~km}$ in along-track coordinates) the ejection velocities are near their maxima.

Takata et al. (1994) modeled the evolution of the plume expansion. They observed a gradual upward ejection of the debris and estimated that more than $40 \%$ of cometary material would rise above the 1 bar level at $100 \mathrm{~s}$ into their simulation, and more than $80 \%$ would eventually ascend above the visible cloud decks. Crawford et al. (1995) calculated that the energy 


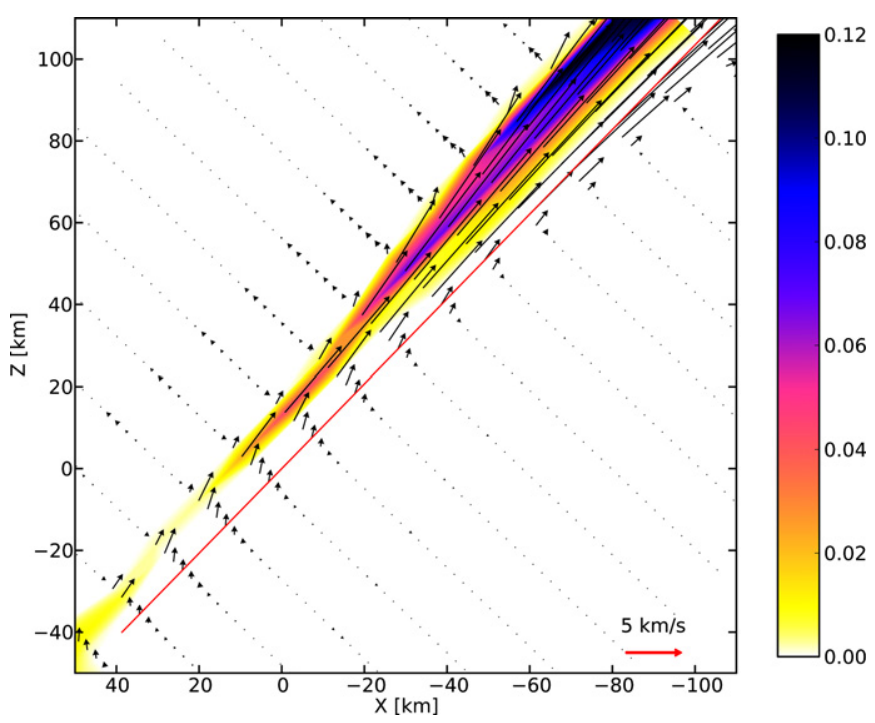

Figure 3. Velocity distribution in our $1 \mathrm{~km}$ diameter impactor model, shown in "true" vertical coordinates. The red line indicates the entry trajectory. Color shows the impactor mass fraction in the ejecta.

(A color version of this figure is available in the online journal.)

and mass deposition of large fragments ( $>1 \mathrm{~km}$ diameter) occurs below Jupiter's ammonia cloud layer and predicted that less than $1 \%$ of the impactor material would be entrained into the rising plume. Lagrangian tracer particles in their model indicated that for a $3 \mathrm{~km}$ diameter impactor the total material in the plume above the 1 bar level is about 6.8 fragment masses, with only $0.2 \%$ being cometary material. Carlson et al. (1997) modeled the mass outflow of the $\mathrm{G}$ impact. They assumed equal Jovian and cometary contributions in the plume, with a total mass of $\sim 2.2 \times 10^{13} \mathrm{~g}$. Based on the chemistry model of Zahnle et al. (1995), they assumed that $40 \%$ of the comet material would produce water, which agrees with the observations by Bjoraker et al. (1996) and Encrenaz et al. (1997) for the G plume splash. At $20 \mathrm{~s}$ into our simulation, the plume is completely detached from the rest of the comet-disturbed material and the mass of the debris that is moving with an upward velocity component of more than $100 \mathrm{~m} \mathrm{~s}^{-1}$ is about $1.6 \times 10^{15} \mathrm{~g}$, more than three times the mass of the original $1 \mathrm{~km}$ diameter fragment. The material with cometary origin in the plume at the same time is about $4.0 \times 10^{11} \mathrm{~g}$, about $0.07 \%$ of the original fragment or about $0.02 \%$ of the total plume material. For the $750 \mathrm{~m}$ and $500 \mathrm{~m}$ diameter impactors the plume masses are $1.1 \times 10^{15} \mathrm{~g}$ and $3.4 \times 10^{14} \mathrm{~g}$, and the comet fractions are $1.4 \times 10^{11} \mathrm{~g}$ and $9.5 \times 10^{10} \mathrm{~g}$, respectively.

Boslough et al. (1995) introduce an upwelling phase. At the depth of maximum energy deposition, a bubble of material forms from cometary material and Jovian air and rises buoyantly. Based on simulations of Crawford et al. (1995), they suggested that the adiabatic expansion of this bubble as it approaches the visible cloud deck is the source for the expanding ring of Hammel et al. (1995). In our $80 \mathrm{~s}$ simulations (not shown), we observe a similar feature forming out of the material below the pinch-off level. We plan experiments to study whether this feature, plume-related shocks, or something else forms the expanding ring. Our simulations indicate that the blowout plume should be relatively water free and most likely it cannot serve as the source of the observed water abundance values (Bjoraker et al. 1996; Encrenaz et al. 1997). Alternatively, this potentially water-rich bubble that forms well below the water cloud level

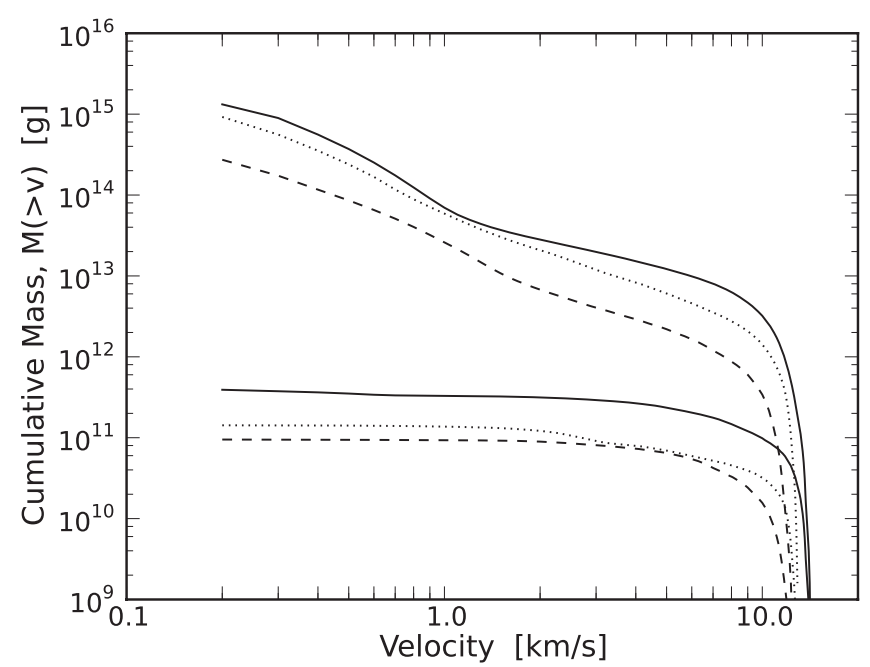

Figure 4. Mass-velocity distribution for $1000 \mathrm{~m}$ (solid), $750 \mathrm{~m}$ (dotted), and $500 \mathrm{~m}$ (dashed) diameter impactors at $20 \mathrm{~s}$. The top curves are for total upwardmoving mass; the bottom curves are for upward-moving comet material only.

and contains most of the comet material could provide an explanation for those observations. There is also the possibility of water forming from other species in the stratosphere and detailed investigation of these hypotheses is needed.

The horizontal ( $x_{1}-x_{2}$ plane) opening angle of the plume cone is $\sim 25^{\circ}$ at the end of our $25-30 \mathrm{~s}$ simulations. This value is lower than expected based on the size of the crescent-shaped debris field. Harrington \& Deming (2001) used a $75^{\circ}$ opening angle to model the crescent and Pankine \& Ingersoll (1999) also stated that the opening angle should be about $70^{\circ}$ to produce a crescent that spans $180^{\circ}$ around the impact site. The smaller opening angle in our model is the result of the plume leaving the computational grid, but we anticipate that the further expansion of the plume at higher altitudes will allow it to open more and we will model this by remapping the variables onto a larger grid. At $30 \mathrm{~s}$ in our simulation, the small amount of cometary material is limited horizontally to a very narrow trail within the plume. This could lead to a higher abundance of cometary material in the middle of the crescent than toward the sides. We plan to study this in the future.

Zahnle \& Mac Low (1995) calculated mass-velocity distributions (MVDs) from simulations similar to those of Zahnle \& Mac Low (1994). The MVD of ejecta from hypervelocity impacts usually follows a power law that can be written in a cumulative form

$$
M(>v) \propto v^{-\alpha}
$$

where $M(>v)$ is the mass at velocities greater than $v$. Their power-law exponent was $\alpha=1.55$. The conservation laws of energy and momentum constrain $\alpha$ to be $1.0<\alpha<2.0$ (Zel'dovich \& Raizer 2002). Harrington \& Deming (2001) used this distribution in their ballistic Monte Carlo plume model. Pankine \& Ingersoll (1999) used two different MVDs in order to simulate the plume and the resulting debris field. The first distribution was based on isentropic expansion of gas into vacuum following an explosion. The second MVD assumed that all the mass was ejected at the same velocity.

Figure 4 shows the MVDs from our simulations. The results follow power-law curves below and above the cutoff velocity of $\sim 10 \mathrm{~km} \mathrm{~s}^{-1}$. The plume generated by the $1 \mathrm{~km}$ diameter impactor has $\alpha \sim 1.4$; for the $750 \mathrm{~m}$ and $500 \mathrm{~m}$ diameter cases $\alpha \sim 1.6$. For the comet material only (bottom curves), these 
curves are much shallower, indicating that proportionally more impactor material ejects at higher speeds.

\section{CONCLUSIONS}

In this study, we focused on the generation and evolution of the initial plume after a Jovian cometary impact. We found that there is a pinch-off point approximately $50 \mathrm{~km}$ below the 1 bar level in the atmosphere, above which the initial plume begins to form and ascend, with velocities up to $\sim 15 \mathrm{~km} \mathrm{~s}^{-1}$. The initial opening angle of the plume is quite narrow, much smaller than the $70^{\circ}$ found in other models. However, further evolution of the plume as it blows out may change this value. The bulk of the cometary material and kinetic energy penetrates to much greater depths; it rises buoyantly and much slower, and merits our further study. The fast plume contains more than three times the mass of the original impactor but is almost entirely (>99.9\%) composed of material from the Jovian atmosphere, so the observed dark features away from the impact site (e.g., main ring, crescent) should contain almost exclusively processed Jovian air. We will continue to investigate Jovian impacts with the goal of relating their physical properties to the observations.

This research was supported by National Science Foundation Grants AST-0813194 and AST-0964078.

\section{REFERENCES}

Bjoraker, G., Stolovy, S. R., Herter, T. L., Gull, G. E., \& Pirger, B. E. 1996, Icarus, 121,411

Boslough, M. B., Crawford, D. A., Trucano, T. G., \& Robinson, A. C. 1995, Geophys. Res. Lett., 22, 1821
Carlson, R. W., Drossart, P., Encrenaz, Th., Weissman, P. R., Hui, J., \& Segura, M. 1997, Icarus, 128, 251

Chodas, P. W., \& Yeomans, D. K. 1996, in IAU Colloq. 156, The Impact of Comet Schoemaker-Levy 9 on Jupiter, ed. K. S. Noll, H. A. Weaver, \& P. D. Feldman (Cambridge: Cambridge Univ. Press), 1

Crawford, D. A., Boslough, M. B., Trucano, T. G., \& Robinson, A. C. 1995, Int. J. Impact. Eng., 17, 253

Deming, D., \& Harrington, J. 2001, ApJ, 561, 468

Encrenaz, Th., Drossart, P., Carlson, R. W., \& Bjoraker, G. 1997, Planet. Space Sci., 45, 1189

Hammel, H. B., et al. 1995, Science, 267, 1288

Hammel, H. B., et al. 2010, ApJ, 715, L150

Harrington, J., \& Deming, D. 2001, ApJ, 561, 455

Harrington, J., de Pater, I., Brecht, S. H., Deming, D., Meadows, V. S., Zahnle, K., \& Nicholson, P. D. 2004, in Jupiter: The Planet, Satellites, and Magnetosphere, ed. F. Bagenal, T. E. Dowling, \& W. B. McKinnon (Cambridge: Cambridge Univ. Press), 159

Hayes, J. C., Norman, M. L., Fiedler, R. A., Bordner, J. O., Li, P. S., Clark, S. E., ud-Doula, A., \& Mac Low, M.-M. 2006, ApJS, 165, 188

Hueso, R., et al. 2010, ApJ, 721, L129

Jessup, K. L., Clarke, J. T., Ballester, G. E., \& Hammel, H. B. 2000, Icarus, 146, 19

Khokhlov, A. 1994, ApJ, 124, L115

Korycansky, D. G., Harrington, J., Deming, D., \& Kulick, M. E. 2006, ApJ, 646, 642

Korycansky, D. G., Zahnle, K. J., \& Mac Low, M.-M. 2002, Icarus, 157, 1

Mac Low, M.-M., \& Zahnle, K. 1994, ApJ, 434, L33

Pankine, A. A., \& Ingersoll, A. P. 1999, Icarus, 138, 157

Sánchez-Lavega, A., et al. 2010, ApJ, 715, L155

Takata, T., \& Ahrens, T. J. 1997, Icarus, 125, 317

Takata, T., O’Keefe, J. D., Ahrens, T. J., \& Orton, G. S. 1994, Icarus, 109, 3

Zahnle, K., \& Mac Low, M.-M. 1994, Icarus, 108, 1

Zahnle, K., \& Mac Low, M.-M. 1995, J. Geophys. Res., 100, 16885

Zahnle, K., Mac Low, M.-M., Lodders, K., \& Fegley, B., Jr. 1995, Geophys. Res. Lett., 22, 1593

Zel'dovich, Ya. B., \& Raizer, Yu. P. 2002, Physics of Shock Waves and HighTemperature Hydrodynamic Phenomena (Mineola, NY: Dover) 